\title{
DESCRIPTION OF LIGHT ION PRODUCTION CROSS SECTIONS AND FLUXES ON THE MARS SURFACE USING THE QMSFRG MODEL
}

\author{
Francis A. Cucinotta ${ }^{1}$, Myung-Hee Kim ${ }^{1}$, \\ Irene Schneider ${ }^{2}$, and Donald M. Hassler ${ }^{3}$ \\ ${ }^{1}$ NASA, Lyndon B. Johnson Space Center Houston TX, USA \\ ${ }^{2}$ Penn State University, State College, PA, USA \\ ${ }^{3}$ Southwest Research Institute, Boulder, CO, USA
}

\begin{abstract}
The atmosphere of Mars significantly attenuates the heavy ion component of the primary galactic cosmic rays (GCR), however increases the fluence of secondary light ions (neutrons, and hydrogen and helium isotopes) because of particle production processes. We describe results of the quantum multiple scattering fragmentation (QMSFRG) model for the production of light nuclei through the distinct mechanisms of nuclear abrasion and ablation, coalescence, and cluster knockout. The QMSFRG model is shown to be in excellent agreement with available experimental data for nuclear fragmentation cross sections. We use the QMSFRG model and the space radiation transport code, HZETRN to make predictions of the light particle environment on the Martian surface at solar minimum and maximum. The radiation assessment detector (RAD) experiment will be launched in 2009 as part of the Mars Science Laboratory (MSL). We make predictions of the expected results for time dependent count-rates to be observed by RAD experiment. Finally, we consider sensitivity assessments of the impact of the Martian atmospheric composition on particle fluxes at the surface.
\end{abstract}




\section{INTRODUCTION}

Human beings are scheduled to visit Mars in the 2030 to 2040 time-period. Prior to their arrival an accurate assessment of the radiation environment on the Mars surface is required. Particle flux distributions, which describe particle type (charge and mass), $j$, kinetic energy, $E(\mathrm{MeV} / \mathrm{u})$ and particle directions are necessary for a complete description of radiation fields in space because the use of dose equivalent carries large uncertainties in projecting risk of late effects from galactic cosmic rays (GCR) [1-3]. The primary GCR are well described by empirical models based on extensive measurements [4]. The description of the GCR on the planetary surface of Mars is expected is more challenging because of larger amount of material ions traverse in the Mars atmosphere and backscatter

of neutrons and other light ions from the Mars regolith [5]. Nuclear fragmentation and energy loss processes are the main physical mechanisms in GCR transport. In this report we briefly summarize a quantum multiple scattering model of nuclear fragmentation (QMSFRG) and describe its extension to incorporate nuclear coalescence as a mechanism of light ion production. The RAD experiment will be launched in 2009 as part of the Mars Surface Lander (MSL) [6]. In this report we discuss predictions of the expected results for time dependent count-rates to be observed by RAD experiment on Mars, and evaluate the role of the altitude of a landing site and the atmospheric composition.

\section{METHODS}

\section{Quantum Fragmentation Model}

The QMSFRG considers the multiple scattering series for two heavy ions and introduces the impulse and eikonal approximation for the total momentum transfer vector in order to 
obtain a closed-form solution to the abrasion cross section excitation spectrum [7-10]. For inclusive reactions where a single fragment originating in the projectile is measured, closure is performed on the final target state with a momentum vector denoted $\mathbf{p}_{X}$ used to represent these states. The reaction is assumed to proceed through the abrasion step producing a pre-fragment, $F^{*}$ and fireball piece from the projectile-target overlap denoted, $R$, followed by the ablation step where the final projectile fragment, $F$ is formed after nuclear de-excitation. The total momentum transfer is $\mathbf{q}=\mathbf{p}_{T}-\mathbf{p}_{X}$ where $\boldsymbol{p}_{T}$ is the initial target momentum. The pre-fragment, $F^{*}$ excitation spectrum following nucleon or alpha particle abrasion was found [7,8] as an impact parameter dependent convolution of the pre-fragment excitation response for a transition of the pre-fragment core from state $n$ to $n$ ' and the project fireball response, average over the target ground-state wave function, $\mid \mathrm{T}>$,

$$
\frac{d \sigma}{d E_{F^{*}}}=<T\left|\int d^{2} q d^{2} b d^{2} b^{\prime} \quad e^{i \mathbf{q (}\left(\mathbf{b}-\mathbf{b}^{\prime}\right)} P_{n, n^{\prime}}\left(b, b^{\prime}, E_{F^{*}}\right) \Lambda_{n, n^{\prime}}\left(q, b, b, E_{F^{*}}\right)\right| T>
$$

where $b\left(b^{\prime}\right)$ is the impact parameter, and $q$ the momentum transfer. The abrasion response is defined as the quantum matrix elements for the interaction of the projectile fireball, $\mathrm{R}$ with the target, $\mathrm{T}$ after performing closure over the final fireball states (denoted $\mid \mathrm{R}>$ )

$$
\Lambda_{n, n^{\prime}}\left(q, b, b^{\prime}, E_{F^{*}}\right)=\int \frac{d \mathbf{k}_{R}}{(2 \pi)^{3}} \quad<R^{\prime}\left|Q_{R T}{ }^{+}\left(b^{\prime}\right)\right| R><R\left|Q_{R T}(b)\right| R^{\prime}>\delta\left(E_{i}-E_{f}\right)
$$

where the $Q_{R T}$ represent the fireball-target profile operator, and $k_{R}$ the projectile fireball momentum vector. The abrasion-response represents a complicated many-body operator that is solved by approximation using closure over the target and fireball states for evaluating the pre-fragment distribution. The one-particle abrasion response has been evaluated using the shell model response functions $[8,9]$. The pre-fragment excitation is described in terms of the transition matrix

$$
P_{n, n^{\prime}}\left(b, b^{\prime}, E_{F^{*}}\right)=<F_{n}^{*}\left|Q_{F^{*} T}^{+}\left(b^{\prime}\right)\right| F_{n^{\prime}}^{*}><F_{n^{\prime}}^{*}\left|Q_{F^{*} T}(b)\right| F_{n}^{*}>
$$


where quantum matrix elements for the pre-fragment excitation are evaluated over the many-body profile operators, $Q_{F^{*} T}$. In the model a convolution approach is used to derive the multi-nucleon abrasion excitation spectrum from the single-fragmentation term $[7,8]$. The resulting excitation spectrum is broad with a shape similar to a log-normal distribution with mean energies from 20 to $30 \mathrm{MeV}$ for one-nucleon removal.

The de-excitation of the pre-fragments in nuclear ablation is described in a stochastic process using a Master equation for de-excitation by particle emission $[9,10]$. If $f_{b}(E, t)$ is the probability of finding the nuclei $b$ at time $t$ with excitation energy $E_{b}$ and $P_{b, k}(E)$ be the probability that the nuclei, $b$ will emit ion $k$ with energy $E$, then the Master equation is

(4) $\frac{d f_{b}\left(E_{b}^{*}, t\right)}{d t}=\sum_{j} \int d E f_{a}\left(E_{a}^{*}, t\right) P_{a, j}(E)-\sum_{k} \int d E f_{b}\left(E_{b}^{*}, t\right) P_{b, k}(E)$

The first-term on the right side of Eq. (4) corresponds to gains by decays of ion $a$ emitting ion $j$ to form ion, $b$, and the second term from losses due to decays of ion $b$ into ion $c$ by emitting ion $k$ where the $j$ (or $k$ ) are light-particle emissions $(n, p, d, t, h$, or $\alpha$ ). The probability of finding the nuclei $b$ at time $t$ with $E^{*}{ }_{b}$ can be divided into stable and unstable parts depending on the lowest excitation energy of $E^{*}{ }_{b}$, denoted $\min \left[S_{b j}\right]$,

$$
f\left(E_{b}^{*}, t\right)=g\left(E_{b}^{*}, t\right)+h\left(E_{b}^{*}, t\right)
$$

where $g$ and $h$ denote the stable and unstable parts of $f$, respectively. As $t \rightarrow \infty$, we have $h \rightarrow 0$ such that

(6) $\lim _{t \rightarrow \infty} f\left(E_{b}^{*}, t\right)=g\left(E_{b}^{*}, \infty\right)$

The probabilities for a single-step decay of nucleus $a$, are defined as

(7) $F_{a, j}=\int_{0}^{E^{*}-S_{a, j}} P_{a, j}(E) d E$ 
with a stable daughter nuclei formed corresponding to the probability

(8) $G_{a, j}=\int_{E^{*}-S_{a, j}-\min \left[S_{b, k}\right]}^{E^{*}-S_{a, j}} P_{a) d E}(E)$

and an unstable daughter nuclei formed with probability

(9) $H_{a, j}=\int_{0}^{E^{*}-S_{a, j}-\min \left[S_{b, k}\right]} P_{a, j}(E) d E$

These probabilities obey

(10) $F_{a, j}=G_{a, j}+H_{a, j}$

and the normalization condition, $\quad \sum_{j} F_{a, j}=1$. Because only stable nuclei remain at long-times, the differo-integral Eq (4) is separated using the above definitions as

(11) $g_{b}\left(E_{b}^{*}, \infty\right)=g_{b}\left(E_{b}^{*}, 0\right)+\sum_{j} \int_{0}^{\infty} d t \int d E f_{a}\left(E_{b}^{*}, t\right) P_{a, j}(E)$

and similarly

$$
0=h_{b}\left(E_{b}^{*}, 0\right)+\sum_{j} \int_{0}^{\infty} d t \int d E f_{a}\left(E_{b}^{*}, t\right) P_{a, j}(E)
$$

In evaluating fragmentation cross sections, equations (11) and (12) are solved by iteration up to excitation energies of $200 \mathrm{MeV}[9,10]$, and using the approximation of Campi and Hufner [11] at higher values. The use of the statistical decay model with accurate nuclear level densities that include nuclear shell structure effects and the use of measured values 
for the nuclear masses are important for accurately predicting the odd-even effects in the fragment spectrum.

To estimate nuclear coalescence contributions to $d, t, h$, and $\alpha$ production we consider the momentum distribution for proton $(p)$ and neutron $(n)$ production in the QMSFRG model $[10,13]$, and apply the usual coalescence model [12], which consists of an $A_{n}$-fold folding of the $\mathrm{p}$ and $\mathrm{n}$ - distribution to form a light ion of mass number $\mathrm{A}_{\mathrm{n}}$. The $n$ and $p$ production cross sections are then reduced by the appropriate balancing of the cross sections resulting in light nuclei coalescence. In these estimates only the contributions from abrasion momentum distribution's is used.

\section{Space Radiation Transport Code}

We use the HZETRN code of Wilson et al. $[14,15]$ in our predictions of the Mars surface. HZETRN solves for the spectrum of heavy ion fragment from projectile and target nuclei in the continuous slowing down and straight-ahead approximations. Integration over the Martian atmospheres is performed as described previously [3]. We have modified the HZETRN code in several ways: First we have extended the ion grid to 190-ions from the earlier 59- [13] or 170-ion [16] grids with the ions selected after studying the fragment spectra for most GCR nuclei for primaries of $Z=1$ to 28 . Second we use the QMSFRG data base as described above. Finally, we use the primary GCR model of Badhwar et al. [4], but re-distribute the most abundant element used in this work amongst the isotopic composition found for the primary GCR as described in Cucinotta et al. [16].

\section{RESULTS AND DISCUSSION}

We first describe comparison of the QMSFRG model to heavy ion fragmentation cross sections. Our recent publication [16] made extensive comparisons of QMSFRG to experimental cross sections for $\mathrm{Ne}, \mathrm{Mg}, \mathrm{Ar}$, and Fe. In Figure 1 we show comparisons of the correlations between QMSFRG and the recent data by Zeitlin et al. [17] for ${ }^{28} \mathrm{Si}$ fragmentation cross sections at several energies for $\mathrm{C}$ and $\mathrm{Al}$ targets. As in our earlier work, QMSFRG demonstrates good agreement with fragmentation cross sections for 
projectile fragments as function of target mass and beam energy with over $85 \%$ of the cross sections agreeing to within \pm 25 percent of measurements. Light ions are produced by several mechanisms including nuclear abrasion, ablation, coalescence, cluster knockout, and electro-magnetic dissociation. In Table 1 we show contributions for several of these mechanisms for ${ }^{16} \mathrm{O}$ fragmentation on several targets and compare to the experimental data of Olsen et al. [18]. Coalescence is predicted to be a dominant mechanism for $\mathrm{d}, \mathrm{t}, \mathrm{h}$, and $\alpha$-particle production. Calculations include cluster knockout only for $\alpha$-particle production and appear to be most important for $\mathrm{H}$ and other light targets. Studies of $\mathrm{d}, \mathrm{t}$, and $\mathrm{h}$ cluster knockout maybe warranted, especially for target fragmentation data bases.

In Table 2 and Figures 2 and 3 we show predictions for the Mars surface. Table 2 shows the elemental fluence at solar minimum where the solar modulation parameter, $\Phi$ of 428 MV is used [4] and for the year 2010 a modulation parameter of $1000 \mathrm{MV}$. The Mars atmosphere significantly depletes the HZE components and the $Z=1,2$ ions will be the main inducers of biological risk for astronauts occupying the Mars surface. The angular view of RAD will be no more than 90 degrees because of the mass limitations on MSL, and we show results for the complete horizon and a restricted vertical horizon of 60 and 90 degrees in Table 2. In Figure 2 we show results for the energy spectra and average fluence of light and heavy ions for different altitudes relative to the mean surface. The shape of the spectrum below $200 \mathrm{MeV} / \mathrm{u}$ changes dramatically due to production from GCR heavy ions or target atoms in the atmosphere. We have not included the backward neutron components in these comparisons, which significantly increase the neutron components below about $100 \mathrm{MeV}$ and can lead to increases on more than one order of magnitude for neutrons below about $10 \mathrm{MeV}$ especially for landing sites on Mars regolith.

The Mars atmosphere is largely $\mathrm{CO}_{2}$, however contains a small amount of other constituents [19-21]. It is also of interest for improved understanding of the possible history of life on Mars to consider the evolution and possible changes of the Martian atmosphere and their impact on radiation effects for possible life in the past. Figure 3 makes predictions for several possible atmospheric conditions including a methane 
atmosphere or mixed $\mathrm{CH}_{2}$ and $\mathrm{CH}_{4}$ atmosphere. Along with the vertical height of the atmosphere, the mass contribution of hydrogen makes the most significant modulation of the surface radiation environment. The relative contributions from non-hydrogen atoms has only a small effect on the charged-particles observed on the Mars surface, however are expected to have a slightly larger dependence for the albedo neutrons [5].

In summary, we have shown that the quantum multiple scattering model of nuclear fragmentation (QMSFRG) provides an accurate data base for GCR transport problem applications. Because the atmosphere of Mars represent one to two mean free paths for GCR heavy ions leading to buildup of light ion fragments, we have made predictions of the flux rates to be expected to be observed by RAD in 2010 for light and heavy particles, which include the nuclear coalescence contribution to the cross sections. Because the integral flux of GCR nuclei changes slowly with atmospheric parameters or geographical location of Mars, measurements of charge-specific energy spectra between 10 and $200 \mathrm{MeV} / \mathrm{u}$ are shown to provide the optimal tests of radiation transport codes. Future work will aim to improve the calculation of cross sections in the coalescence and cluster knockout models $[8,12]$, include the albedo neutron and light ion contributions, and describe detailed geometric models of the RAD configuration on MSL in preparation for the data analysis phase of the experiment.

\section{ACKNOWLEDGEMENTS}

This work was sponsored, in-part, by the NASA Exploration Systems Management Directorates Space Radiation Research Programs Radiation Risk Assessment (F A Cucinotta) and Mars Surface Lander Awards (D Hassler). 


\section{REFERENCES}

1. Cucinotta F A, Durante M (2006) Cancer risks from exposures to galactic cosmic rays: Implications for space exploration by human beings. The Lancet Oncology 7: 431-435.

2. Cucinotta F A, Schimmerling W, Wilson J W, Peterson L E, Badhwar, G D, Saganti P, Dicello J F (2001) Space radiation cancer risks and uncertainties for Mars missions. Radiat Res 156: 682-688.

3. Saganti P B, Cucinotta F A, Wilson J W, Simonsen L C, and Zeitlin C J (2004) Radiation climate map for analyzing risks to astronauts on the Mars surface from galactic cosmic rays. Space Science Rev 110: 143-156.

4. Badhwar G B, Cucinotta F A, and O'Neill P M (1994) An analysis of interplanetary space radiation exposure for various solar cycles. Radiat Res 138: 201-208.

5. Clowdsley M, Wilson J W, Kim M H , Singleterry R C, Tripathi, R K, Heinbockel J H, Badavi F F and Shinn J L (2001) Neutron environments on the Martian surface. Phys Medica XVII: 94-96.

6. Hassler,

7. Cucinotta F A, Townsend L W, Wilson J W (1992) Multiple scattering effects in quasielastic $\alpha-{ }^{4} \mathrm{He}$ scattering. Phys Rev C46: 1451-1456.

8. Cucinotta F A, Dubey R R (1994) Alpha cluster description of excitation energies in ${ }^{12} \mathrm{C}\left({ }^{12} \mathrm{C}, 3 \alpha\right) \mathrm{X}$ at $2.1 \mathrm{GeV}$. Phys Rev C50: 979-984.

9. Cucinotta F A, et al (1997) Computational procedures and data-base development. In: NASA Workshop on Shielding Strategies for Human Space Exploration. Eds. Wilson JW, Miller J, Konradi A, and Cucinotta FA. NASA CP-3360.

10. Cucinotta F A, Wilson J W, Townsend L W (1997) Abrasion-ablation model for neutron production in heavy ion collisions. Nucl Phys A. 619: 202-212.

11. Campi X, and Hufner J (1981) Nuclear spallation-fragmentation reactions induced by high-energy projectiles. Phys Rev C24: 2199-2209.

12. Leupold S, and Heinz U (1994) Coalescence model for deuterons and anti-deuterons in relativistic heavy-heavy ion collisions. Phys Rev C50: 1110-1128.

13. Cucinotta F A (1994) Forward production of protons in relativistic ${ }^{12} \mathrm{C}$-nucleus collisions. J Phys G: Nucl Part Phys 20: 1803-1815.

14. Wilson J W, Badavi F F (1986) Methods of galactic heavy ion transport. Radiat Res 108: 231-237. 
15. Wilson J W, Townsend L W, Schimmerling W, Khandelwal G S, Khan F, Nealy J E, Cucinotta F A, Simonsen L C, Shinn J L, Norbury J W (1991) Transport methods and interactions for space radiations. NASA RP-1257.

16. Cucinotta F A, Wilson J W, Saganti P, Hu X, Kim M Y, Cleghorn T, Zeitlin C, and Tripathi R K (2006) Isotopic dependence of GCR fluence behind shielding. Rad Measurements (in press).

17. Zeitlin C, Fukumura A, Guetersloh S B, Heilbronn L H, Iwata Y, Miller J, and Murakami T (2006) Fragmentation cross sections of ${ }^{28} \mathrm{Si}$ at beam energies from $290 \mathrm{~A}$ to $1200 \mathrm{~A} \mathrm{MeV}$. Nucl Phys A (in Press).

18. Olsen D L, Berman B L, Greiner D E, Heckman H H, Lindstrom P J, Crawford H J (1981) Factorization of fragment-production cross sections in relativistic heavy-ion collisions. Phys Rev C28: 1602-1613.

19. Mumma M J, Novak R E, DiSanti M A, and Bonev, B P (2003) A sensitive search for methane on Mars (abstract). $35^{\text {th }}$ Annual DPS Meeting, Monterey, California.

20. Krasnopolsky V A, Maillard J P, and Owen T C (2004) Detection of methane in the martian atmosphere: evidence for life? Icarus 172: 537-547.

21. Formisano V, Atreya S, Encrenaz T, Ignatiev N, and Giuranna M (2004) Detection of methane in the atmosphere of Mars. Science 306: 1758-1761. 


\section{FIGURE CAPTIONS}

Figure 1. Comparisons of fragmentation cross sections in QMSFRG model to experimental data [19] for ${ }^{28} \mathrm{Si}$ fragmentation on $\mathrm{C}$ and $\mathrm{Al}$ targets at several energies.

Figure 2. Upper panel shows calculations of the energy spectra of $Z=1$ and 2 ions incident on Mars and the average mean altitude projected for 2010 ( $\Phi=1000 \mathrm{MV})$. The lower panel shows calculations of the total annual flux of $Z=0,1,2,6$, and 8 ions for different mean altitudes on Mars projected for $2010(\Phi=1000 \mathrm{MV})$.

Figure 3. Calculations of the dependence of the Mars surface point dose equivalent on atmospheric composition and density. Different atmospheric mixtures are shown including the temptative detection of methane $\left(\mathrm{CH}_{4}\right)$, which vary between $10 \mathrm{ppb}$ (parts per billion) and a maximum of 300ppb of $\mathrm{CH}_{4}$. Two theoretical end cases of a pure $\mathrm{CO}_{2}$ and pure $\mathrm{CH}_{4}$ atmosphere are shown for reference.. 

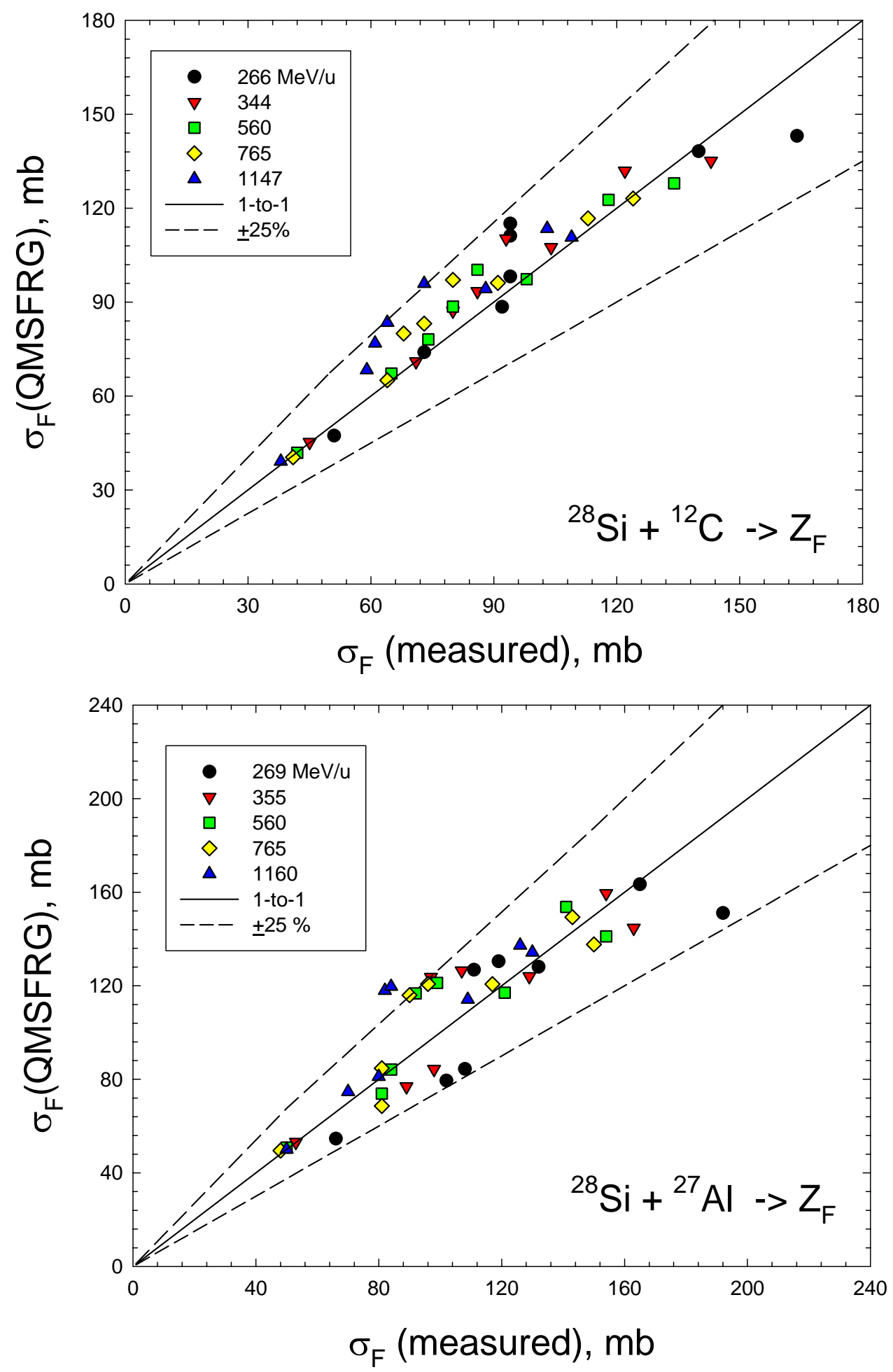

Figure 1. 

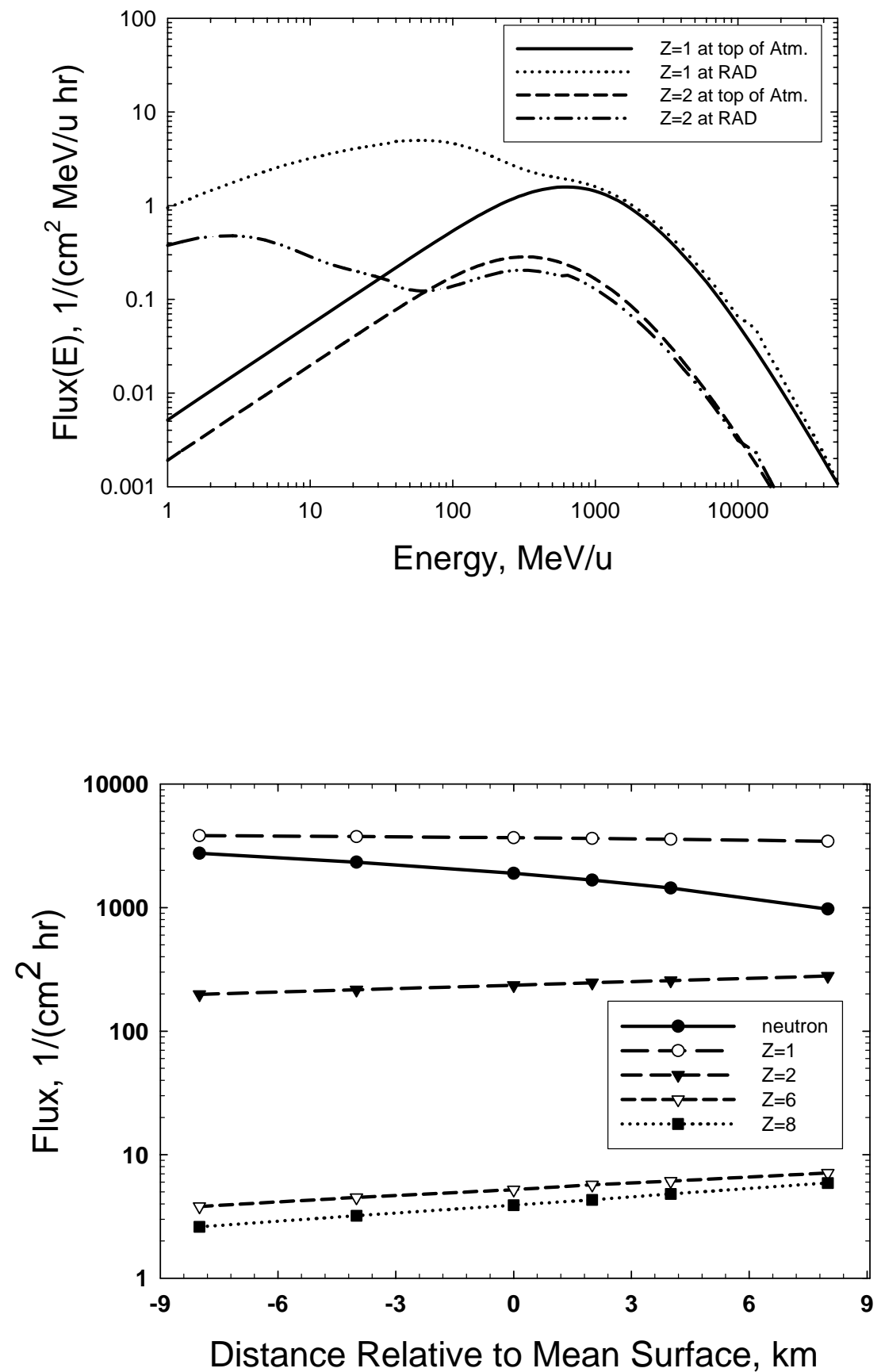

Figure 2 


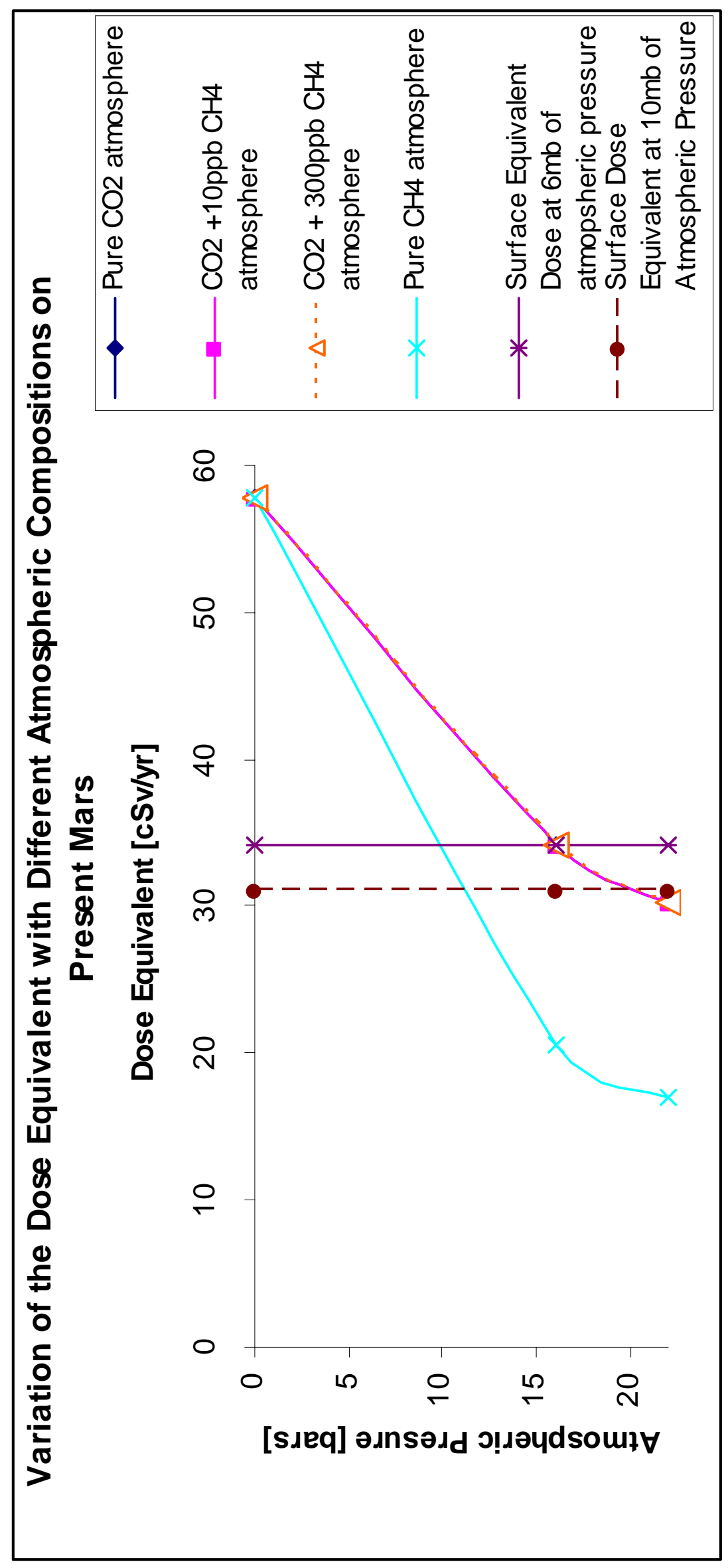

ֻ 

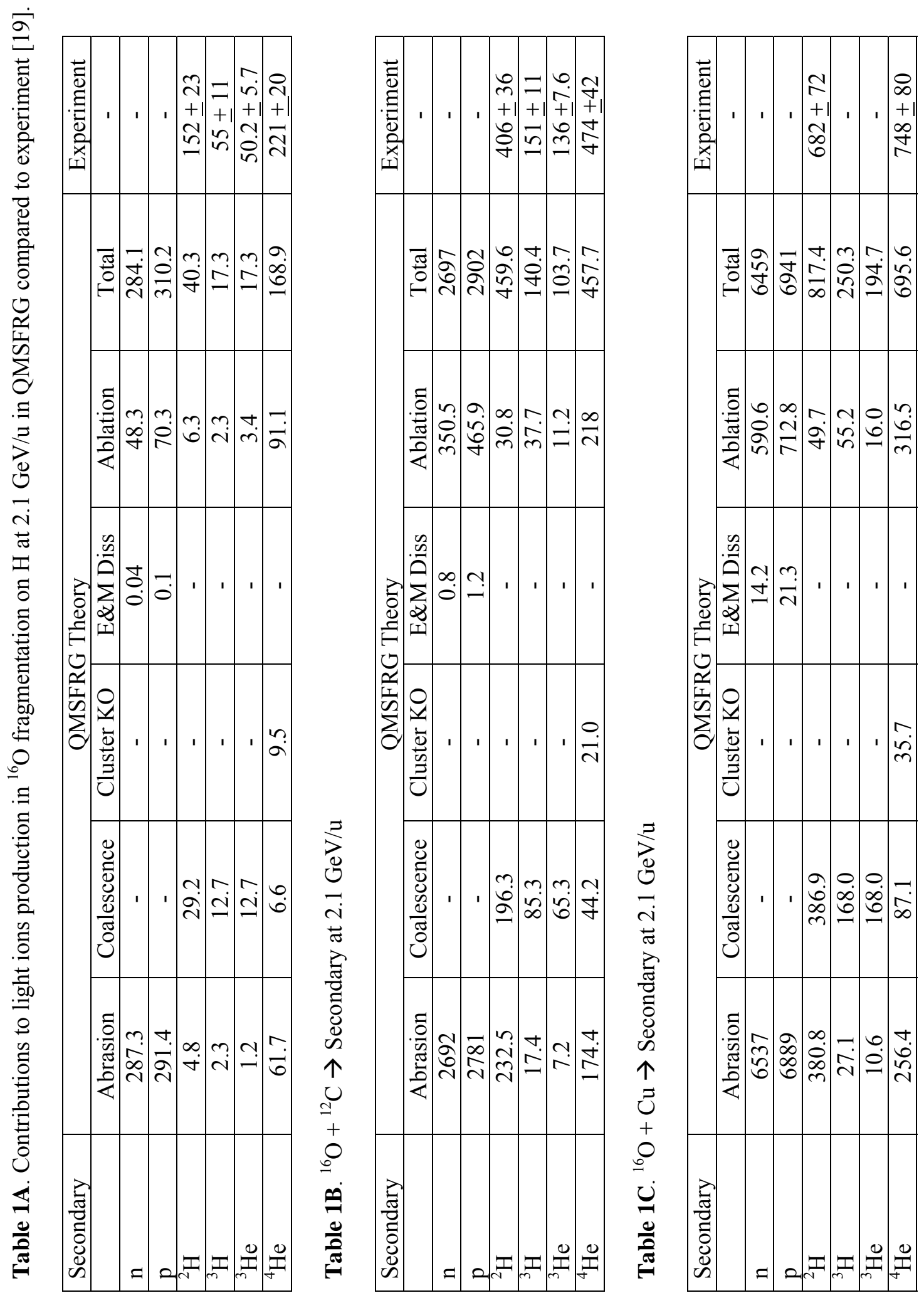

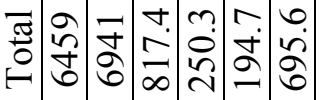

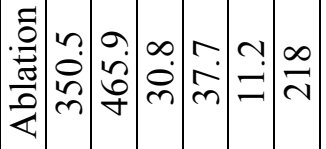

.0ี

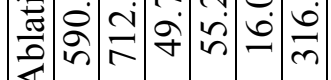

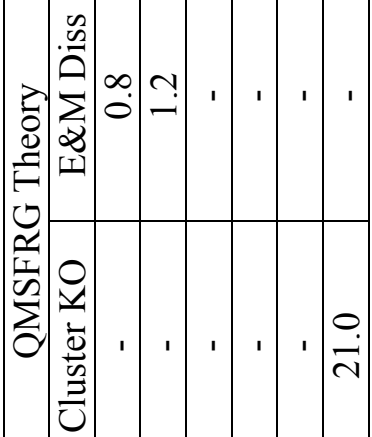

.$\tilde{n}$

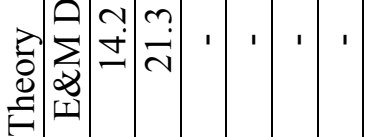

可项

잔.

$\stackrel{\underline{T}}{\infty}$

$\frac{0}{2}$

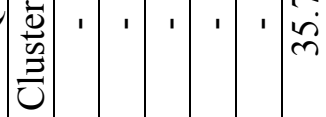

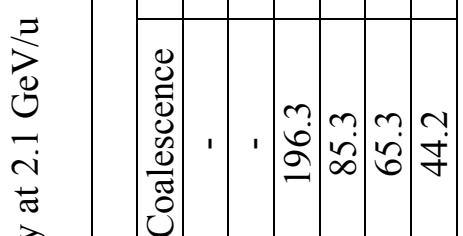

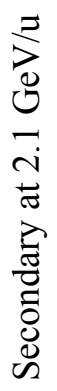

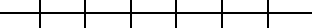

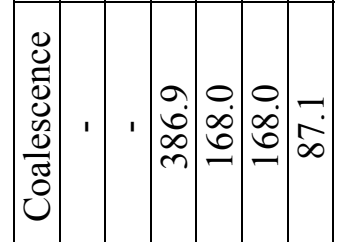

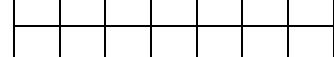

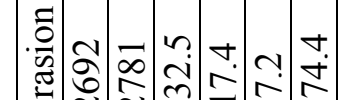

.

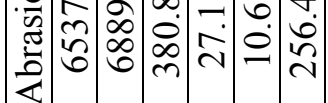

1
0
0
0
0

$\uparrow$
$\Xi$
+
0

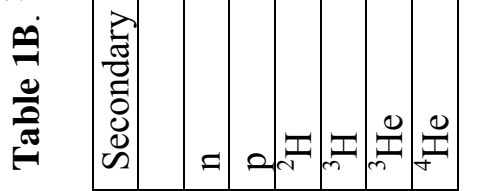

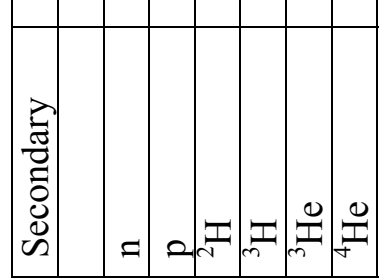


Table 2a. Projections of Elemental GCR fluences for RAD Surface operations in 2010 $(\Phi=1000 \mathrm{MV})$.

\begin{tabular}{|c|c|c|c|}
\hline Charge Group, Z & $\begin{array}{c}\text { Fluence, } 1 /\left(\mathrm{cm}^{2} \mathrm{hr}\right) \\
\text { All angles }\end{array}$ & $\begin{array}{c}\text { Fluence, } 1 /\left(\mathrm{cm}^{2} \mathrm{hr}\right) \\
90 \text { degree viewing } \\
\text { cone }\end{array}$ & $\begin{array}{c}\text { Fluence, } 1 /\left(\mathrm{cm}^{2} \mathrm{hr}\right) \\
60 \text { degree viewing } \\
\text { cone }\end{array}$ \\
\hline 0 & 13841 & 4374 & 2751 \\
\hline 1 & 11576 & 5756 & 3817 \\
\hline 2 & 425 & 288 & 198 \\
\hline 3 & 3.32 & 2.30 & 1.58 \\
\hline 4 & 2.40 & 1.66 & 1.14 \\
\hline 5 & 3.23 & 2.38 & 1.66 \\
\hline 6 & 7.08 & 5.40 & 3.82 \\
\hline 7 & 2.34 & 1.77 & 1.25 \\
\hline 8 & 4.46 & 3.56 & 2.56 \\
\hline 9 & 0.30 & 0.23 & 0.16 \\
\hline 10 & 0.80 & 0.64 & 0.46 \\
\hline 11 & 0.31 & 0.24 & 0.17 \\
\hline 12 & 0.78 & 0.64 & 0.46 \\
\hline 13 & 0.20 & 0.16 & 0.12 \\
\hline $14-17$ & 0.42 & 0.35 & 0.26 \\
\hline $18-23$ & 0.23 & 0.19 & 0.14 \\
\hline$>23$ & 0.18 & 0.16 & 0.12 \\
\hline
\end{tabular}

Table 2b. Same as Fig 2a near Solar Minimum ( $\Phi=428 \mathrm{MV})$

\begin{tabular}{|c|c|c|c|}
\hline Charge Group, Z & $\begin{array}{c}\text { Fluence, } 1 /\left(\mathrm{cm}^{2} \mathrm{hr}\right) \\
\text { All angles }\end{array}$ & $\begin{array}{c}\text { Fluence, } 1 /\left(\mathrm{cm}^{2} \mathrm{hr}\right) \\
90 \text { degree viewing } \\
\text { cone }\end{array}$ & $\begin{array}{c}\text { Fluence, } 1 /\left(\mathrm{cm}^{2} \mathrm{hr}\right) \\
60 \text { degree viewing } \\
\text { cone }\end{array}$ \\
\hline 0 & 24779 & 8131 & 5127 \\
\hline 1 & 22552 & 11711 & 780 \\
\hline 2 & 770 & 527 & 364 \\
\hline 3 & 5.94 & 4.19 & 2.89 \\
\hline 4 & 4.20 & 2.96 & 2.04 \\
\hline 5 & 5.58 & 4.17 & 2.93 \\
\hline 6 & 12.09 & 9.37 & 6.67 \\
\hline 7 & 3.89 & 2.99 & 2.12 \\
\hline 8 & 7.40 & 5.99 & 4.34 \\
\hline 9 & 0.48 & 0.36 & 0.26 \\
\hline 10 & 1.25 & 1.01 & 0.73 \\
\hline 11 & 0.48 & 0.38 & 0.27 \\
\hline 12 & 1.19 & 0.99 & 0.72 \\
\hline 13 & 0.31 & 0.25 & 0.18 \\
\hline $14-17$ & 0.96 & 0.80 & 0.59 \\
\hline $18-23$ & 0.33 & 0.28 & 0.20 \\
\hline$>23$ & 0.25 & 0.22 & 0.17 \\
\hline
\end{tabular}

